\title{
Experimental study of three-nucleon dynamics in the dp breakup collisions using the WASA detector
}

\author{
B. Kłos ${ }^{1, \text { a }}$, I. Ciepał ${ }^{2}$, G. Khatri ${ }^{3}$, St. Kistryn ${ }^{3}$, A. Kozela ${ }^{2}$, A. Magiera ${ }^{3}$, W. Parol ${ }^{3}$, I. Skwira- \\ Chalot $^{4}$, and E. Stephan ${ }^{1}$ \\ for the WASA-at-COSY Collaboration
}

${ }^{1}$ University of Silesia, PL-40007 Katowice, Poland

${ }^{2}$ Institute of Nuclear Physics PAN, PL-31342 Kraków, Poland

${ }^{3}$ Jagiellonian University, PL-30059 Kraków, Poland

${ }^{4}$ Faculty of Physics, University of Warsaw, Warsaw, Poland

\begin{abstract}
An experiment to investigate the ${ }^{1} \mathrm{H}(\mathrm{d}, \mathrm{pp}) \mathrm{n}$ breakup reaction using a deuteron beam of 340, 380 and $400 \mathrm{MeV}$ and the WASA detector has been performed at the Cooler Synchrotron COSY-Jülich. The studied energy region, below but close to a pion production threshold, may provide information on various aspects of nuclear interactions, in particular on relativistic effects and their interplay with the three nucleon force (3NF). The almost $4 \pi$ geometry of the WASA detector gives an unique possibility to study the different aspects of nucleon-nucleon dynamics in the three nucleon system. The main steps of the analysis, including energy calibration, PID and studied of efficiency, and their impact on final accuracy of the result, will be discussed.
\end{abstract}

\section{Introduction}

Few-nucleon system is an ideal laboratory to study nuclear forces. The system composed of three nucleons $(3 \mathrm{~N})$ is the simplest nontrivial environment, in which various models of the nucleon-nucleon (NN) interaction can be tested. Three-nucleon system dynamics can be investigated quantitatively by comparing observables calculated with the use of Faddeev equations with precise measurements. The breakup observables can be calculated using modern realistic pairwise nucleon-nucleon NN interactions, combined with model of $3 \mathrm{~N}$ forces [1]. Moreover, the two- and three-nucleon interactions can be modeled within the coupled-channel (CC) framework by an explicit treatment of the $\Delta$-isobar [2]. Alternatively, the dynamics is generated by the chiral effective field theory ( $\chi$ EFT), so far at the next-to-next-to-leading order with all relevant $\mathrm{NN}$ and $3 \mathrm{~N}$ contributions taken into account [3]. The new, improved version of ChPT is currently being developed [4]. The modern theoretical calculations include different pieces of nucleon-nucleon dynamics like the above mentioned three nucleon force but also the long-range Coulomb interaction or relativistic effects. In recent years the relativistic treatment of the breakup reaction in the $3 \mathrm{~N}$ system has been developed using the NN potential [5] and this approach has also been extended for calculations including 3NF [6]. It was shown that in some particular regions of the breakup phase space, relativistic effects can increase or decrease the

\footnotetext{
a e-mail: barbara.klos@us.edu.pl
} 
calculated breakup cross sections by up to $60 \%$. At the same time the effects of $3 \mathrm{NF}$ may change certain observables by a similar factor. The relativistic effects and their interplay with $3 \mathrm{NF}$ become more important with increasing available energy in the three nucleon system. Therefore the energy range of interest is possibly close to pion production threshold (400 $\mathrm{MeV}$ for dp breakup reaction). We measured the differential cross section of the ${ }^{1} \mathrm{H}(d, p p) n$ breakup reaction at beam energies of 340 , 380 and $400 \mathrm{MeV}$. The investigations will enable to study the evolution of the relativistic and $3 \mathrm{NF}$ effects in this energy, possibly setting constraints on the theoretical calculations.

\section{Experiment}

The experiment using the ${ }^{1} \mathrm{H}(\vec{d}, p p) n$ breakup reaction at $340 \mathrm{MeV}, 380 \mathrm{MeV}$ and $400 \mathrm{MeV}$ deuteron beam energy has been performed in January 2013 at the Cooler Synchrotron COSY-Jülich with the WASA-at-COSY detector [7], [8]. The WASA (Wide Angle Shower Apparatus) detector, covering almost full solid angle, gives a unique possibility of complex studies of the dp breakup reaction and the effects beyond non-relativistic NN-interactions, which appear in different phase-space regions. The WASA detector consists of four main components: Central Detector (CD), Forward Detector (FD), Pellet Target Device and the Scattering Chamber.

\section{Data Analysis}

Currently, the data analysis is focused on the proton-proton coincidences registered in the Forward Detector. It aims to determine the differential cross sections on dense angular grid of kinematical configurations defined by the emission angles of the two outgoing protons: two polar angles $\theta_{1}$ and $\theta_{2}$ (in the range between $4^{\circ}$ and $18^{\circ}$ ) and the relative azimuthal angle $\phi_{12}$.

The first step of data analysis is the identification of events of interest, i.e. two protons from the breakup process and deuteron-proton pairs from elastic scattering channel in the Forward Detector. The particle identification is based on the $\Delta E-E$ technique .

The luminosity is determined on the basis of the number of the elastically scattered deuterons at a given $\theta$ angle and on the known cross section for elastic scattering at the studied energy [10]. After selection of deuterons registered in the Forward Detector and energy calibration, the correct energy vs angle relation for elastically scattered deuterons was obtained, see Fig. 1.

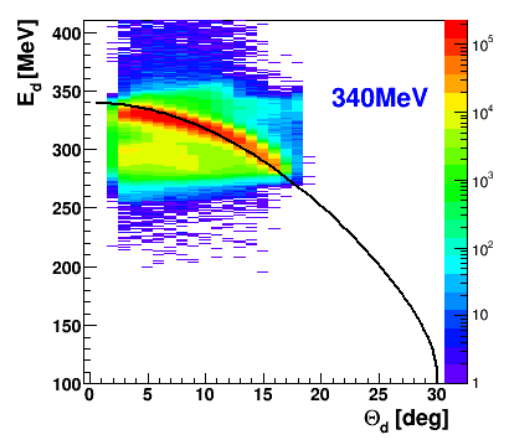

Figure 1. Selection of deuterons registered in FD. Solid curve corresponds to kinematics of elastically scattered deuterons.

After selection of the proton-proton coincidences and having performed the energy calibration, the differential cross section of the breakup reaction can be determined for a chosen kinematic configuration. The configuration is defined by the emission angles of the two outgoing protons: two polar 
angles $\theta_{1}$ and $\theta_{2}$ and the relative azimuthal angle $\phi_{12}$, with defined integration limits: $\Delta \theta_{1}=\Delta \theta_{2}=1^{\circ}$ and $\Delta \phi_{12}=5^{\circ}$. The kinematical spectra $E_{1}$ vs $E_{2}$ are produced for each analyzed configuration.

Events belonging to the chosen configuration of interest are projected onto a kinematical curve in order to obtain the distribution as a function of the arc-length $\mathrm{S}$ measured along the kinematics. An example of the breakup event rate obtained for the chosen kinematical configurations is presented in Fig. 2. The normalization is preliminary: the analysis is ongoing to determine systematic uncertainties related to detection efficiency and to luminosity value obtained on the basis of existing data. It is clear that a very good control over data normalization is indispensable to conclude about relativistic effects.

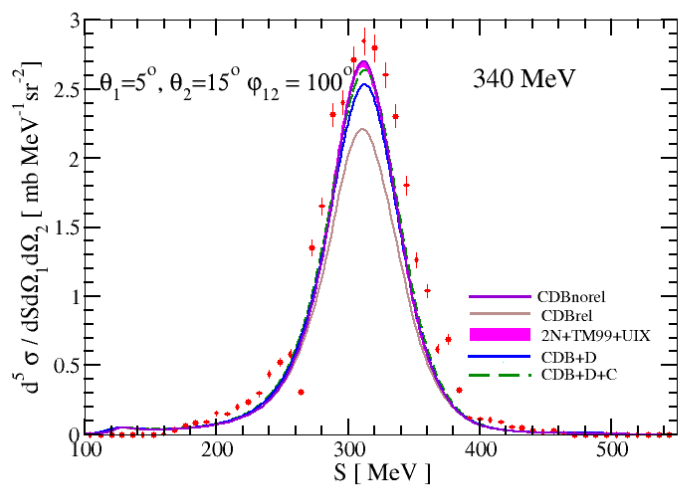

Figure 2. An example of the differential cross section of breakup reaction obtained for chosen kinematical configuration as a function of the $S$ value. Magenta band represents calculations based on realistic potentials: $2 \mathrm{~N}$ complemented with the TM99 $3 \mathrm{NF}$ and the realistic AV18 potential combined with the Urbana IX. The calculations within the coupled-channel approach with the CD Bonn $+\Delta$ potential without and with the Coulomb force included are represented by blue solid line and green dashed line, respectively. Violet and brown solid lines present theoretical calculations with CD Bonn potential in norelativistic and relativistic regime, respectively.

In this figure the theoretical calculations [1] [9] are also shown. The calculations in relativistic regime have recently been performed for the ${ }^{1} \mathrm{H}(d, p p)$ n breakup reaction at the beam energies of 340 , 380 and $400 \mathrm{MeV}$ [11], clearly indicating of relativistic description at these energies.

\section{Summary and outlook}

The analysis is continued with the aim to determine the differential cross sections for the the deuteron breakup process in the $d+p$ system at energies of 340,380 and $400 \mathrm{MeV}$, performed for a large set of kinematical configurations covering a significant part pf the reaction phase space. The data will be compared to the theoretical predictions for three nucleon systems with the aim to investigate relativistic effects. Currently, the analysis is focused on precise determination of efficiency of the detection system and its impact on final accuracy of the result, as well as on deterimination of systematic uncertainty of luminosity. 


\section{Acknowledgements}

This work has been supported by FFE funds of the Forschunszentrum Jülich; by the European Commision under the 7th Framework Programme through the "Research Infrastructures" action of the Capacities Programme (Call: FP7-INFRASTRUCTURES-2008-1) and by Polish National Science Center from grant DEC2012/05/B/ST2/02556.

\section{References}

[1] W. Glöckle, H. Witała, D. Hüber, H. Kamada, J. Golak, Phys. Rep. 274, 107 (1996)

[2] A. Deltuva, K. Chmielewski and P.U. Sauer, Phys. Rev. C 67, 034001 (2003);

A. Deltuva, R. Machleidt and P.U. Sauer, Phys. Rev. C 68, 024005 (2003)

[3] E. Epelbaum, Rep. Prog. Nucl. Phys. 57, 654 (2006)

[4] R. Machleidt and F. Sammarruca, Phys. Scripta 91, 083007 (2016);

D. Djukanovic, E. Epelbaum, J. Gegelia, H. Krebs and U. G. Meißner, Eur. Phys. J. A 51, 101 (2015);

E. Epelbaum, H. Krebs and U. G. Meißner, Phys. Rev. Lett. 115, 122301 (2015);

S. Binder et al., Phys. Rev. C 93, 044002 (2016)

[5] H. Witała, R. Skibiński and J. Golak, The European Physical Journal A 30, 369 (2006)

[6] H. Witała et al., Phys. Rev. C 83, 044001 (2011)

[7] Ch. Bargholtz et al., Nucl. Instrum. Meth. A 594, 339 (2008)

[8] H. H. Adam et al., arXiv:nucl-ex/0411038 (2004)

[9] A. Deltuva et al., Phys. Rev. C 72, 054004 (2005)

[10] K. Ermisch et al., Phys. Rev. C 68, 051001(R) (2003)

[11] H. Witała, privite communication. 